\title{
The Newly Upgraded Large COMPASS Polarized Target
}

\author{
F. Gautheron \\ Physics department, University of Bielefeld, Bielefeld, Germany \\ ON BEHALF OF THE COMPASS COLLABORATION
}

\begin{abstract}
During the CERN SPS 2005 shutdown the COMPASS target system received a major hardware upgrade for the new period of data taking starting in 2006. A new superconducting magnet with a larger acceptance combined with a new microwave cavity and a three cell target setup have been installed and already showed excellent performances that we present for the first time.
\end{abstract}

Keywords: COMPASS, polarized target, ${ }^{6} \mathrm{LiD}$, superconducting magnet

PACS: 29.25.Pj;

\section{INTRODUCTION}

The aim of the COMPASS muon program [1] is to determine how much the gluon contributes to the nucleon polarization. For the measurement of the gluon polarization $\Delta G / G$ the experiment is mainly using the spin asymmetries in the open charm leptoproduction channel and in the high $P_{T}$ hadron pairs channel which are well known both for their sensitivity to $\Delta G$ in deep inelastic muon scattering process. A larger acceptance magnet system was installed in replacement of the previous 69 mrad acceptance one. This superconducting magnet used to polarize the target was commissioned during SPS shutdown in 2005. In the same time a new microwave cavity matching this larger acceptance and a new 3-cell target have also been constructed and installed (Fig. 1).

\section{THE LARGE ACCEPTANCE MAGNET SYSTEM}

A large acceptance $180 \mathrm{mrad}, 2 \mathrm{~m}$ long, solenoid magnet built by Oxford Danfysik ${ }^{1}$ (OD) was delivered at CEA Saclay at the end of 2004 in order to be tested. In February 2005 a cold leak appeared during the first cool down implying the opening of the vacuum vessel to repair a faulty weld. In April 2005 the magnet was energized for the first time at nominal field $(2.5 \mathrm{~T})$ and a quench occured which damaged one of the 16 correction coils ("G2") by developing a superconducting short circuit. The strong coupling between the shorted correction coil G2 and the solenoid is such that each time the solenoid field or the other correction fields are modified, G2 gets loaded by induced high current which may trigger the magnet safety system (MSS) to start a fast discharge. Unfortunately this correction coil could not be repaired in a delay compatible with the operation of

${ }^{1}$ Ferry mills, Osney Mead, Oxford, Oxfordshire,OX2 0ES, UK 


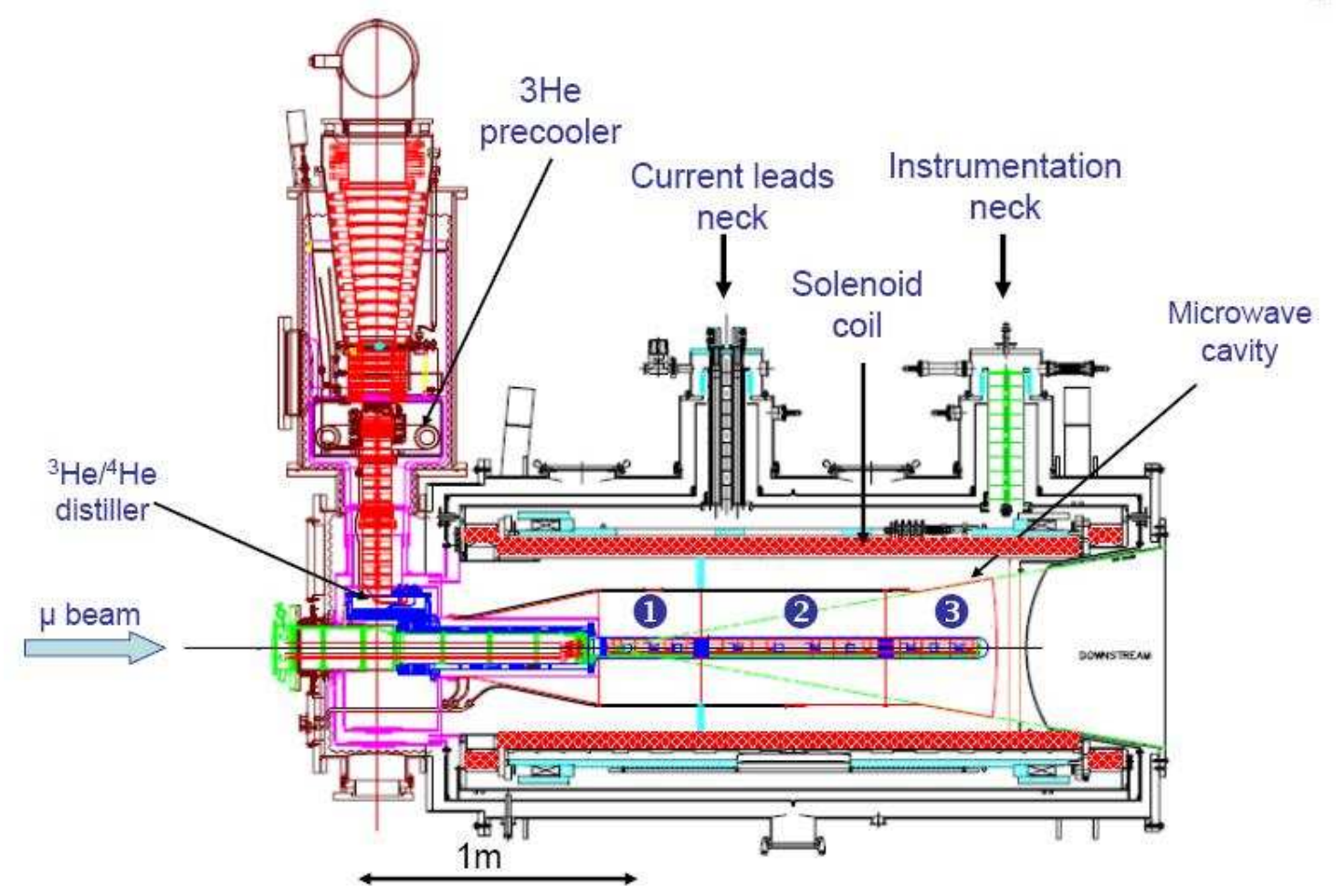

FIGURE 1. Side view of the upgraded COMPASS polarized target.(1), (2) and (3) correspond to the upstream, central and downstream cell respectively

the magnet in 2006. A special procedure was implemented to operate the magnet in a reliable way with a fully reproducible and excellent field homogeneity as required by the dynamic nuclear polarization (DNP) [2] and NMR [3] techniques in order to reach uniform polarization and to measure it accurately.

This procedure took advantage of the heating system installed to protect against quench incidents. To prevent G2 to trap current one switches on the correction coil's heaters to get it resistive. Then the solenoid field can start to be ramped up. After 10 seconds, heaters are turned off and the induced current in G2 is high enough to keep it resistive. A special care has to be taken also concerning the slope of the current ramping during the operation. This procedure made of a succession of perfectly synchronized elementary operations was implemented in the slow control without which the magnet operation would be nearly impossible [4].

We then investigated the homogeneity expected to be better than $10^{-4}$ in the region of the target cells i.e. $\pm 65 \mathrm{~cm}$ along the magnetic axis around the central point of the solenoid. A dedicated bench for magnetic field measurements was built at Saclay consisting of 3 probes (Hall probe, flux coil and NMR probe) moved together along a support with DAQ and displacement fully automated. The profile of each correction coils as well as of the solenoid were determined. An accurate data processing allowed us to extract a single set of coefficients to reach a field homogeneity as good as $3.10^{-5}$ as shown in Fig. 2. 


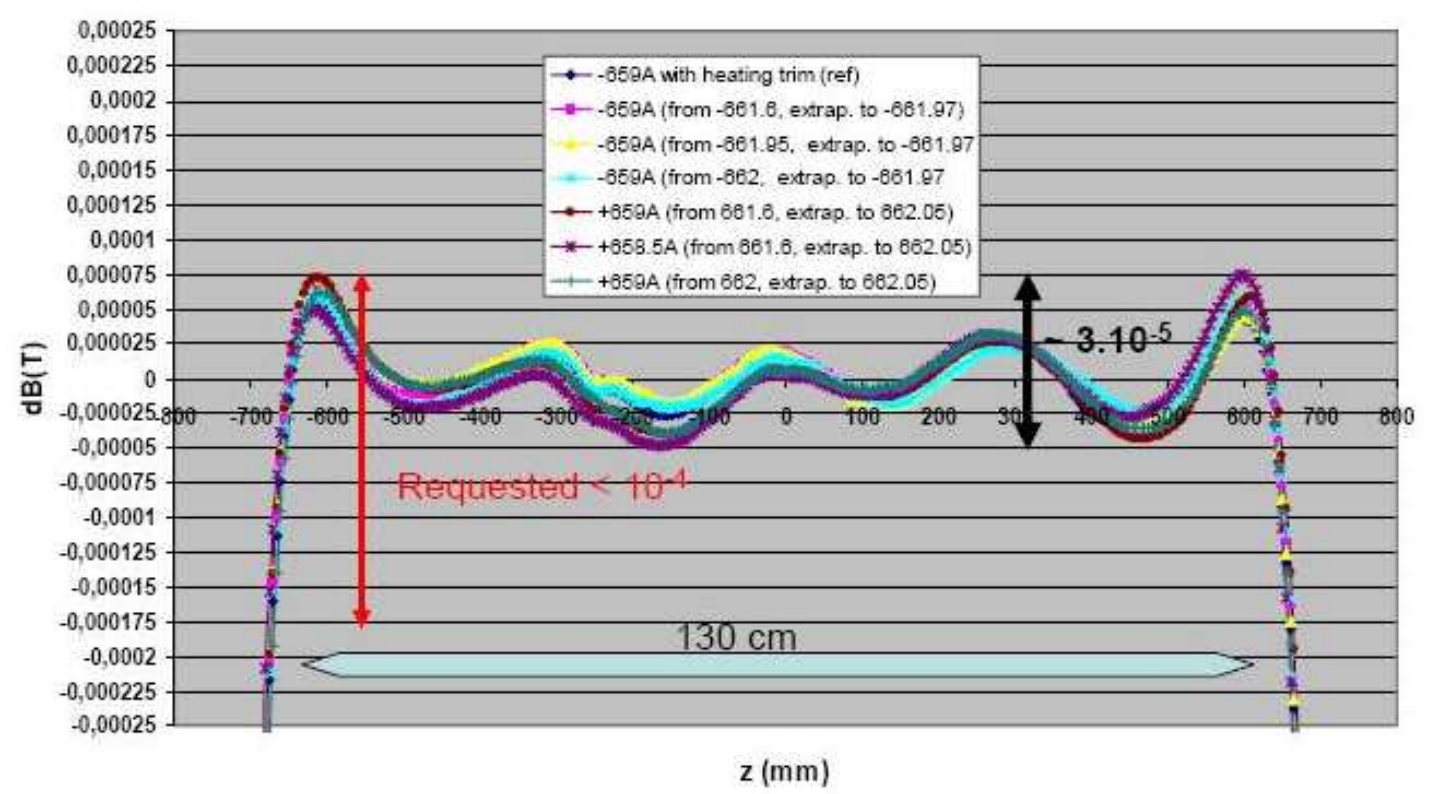

FIGURE 2. Homogeneized solenoid profile at the nominal field of 2.5T. An homogeneity of $\approx 10^{-4}$ was the minimal request and one could reach $3.10^{-5}$

Fulfilling the specifications, the superconducting magnet was then shipped to CERN for installation in the COMPASS hall. The magnetic field profile was remeasured in situ since the location of the magnet is in the vicinity of the first spectrometer dipole magnet ("SM1"). The strong fringe field due to the large acceptance magnetizes the SM1 iron yoke which modifies in turn the solenoid field profile. Using the same bench to determine this influence, a corresponding set of currents for the correction coils was defined with which one reaches an homogeneity of $\pm 410^{-5}$ over the target volume and efficient in both field orientations.

The tests of field rotation were performed during the magnet commissioning and a vertical displacement of about $2 \mathrm{~mm}$ was measured due to the strong torque from the coupling between the field of SM1 and the solenoid fringe field. To reduce this movement the COMPASS collaboration took the decision to operate the solenoid magnet at $1 \mathrm{~T}$ instead of $2.5 \mathrm{~T}$ once the target material had been polarized. One of the $10 \mathrm{NMR}$ coils was dedicately tuned to monitor the evolution of the polarization at $1 \mathrm{~T}$ in the frozen spin mode.

\section{THE 3-CELL TARGET SETUP AND THE NEW MICROWAVE CAVITY}

The COMPASS experiment had been running from 2001 to 2004 with 2 oppositely polarized $60 \mathrm{~cm}$ long target cells (Fig. 3a) and the polarization was reversed by rotation of the magnetic field. Thus, the spin orientation for both cells stays constant with respect to the field orientation which is a source of false asymmetries which one tries to get rid 
a)
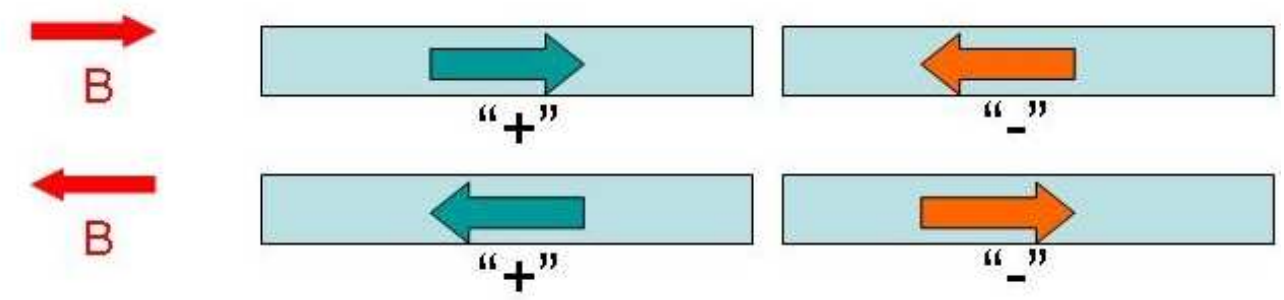

b)
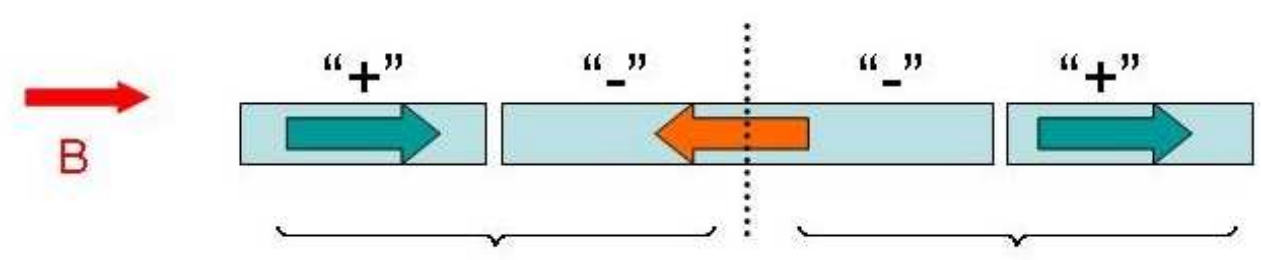

FIGURE 3. Previous target cell configuration used from 2001 to 2004 (a) and new 3 cells configuration since 2006 (b)

of as much as possible. The spin direction can be inverted with respect to the magnetic field using the microwave reversal procedure. This was performed once or twice a run ( 6 month) every year of data taking because a high degree of polarization $(>50 \%)$ is only reachable after several days of DNP for ${ }^{6} \mathrm{LiD}$, the target material. Within these periods, the efficiency of the spectrometer is not necessarily the same in the 2 opposite configurations periods because the properties of the detectors may have drifted. The simulation of a 3-cell $(30 \mathrm{~cm} / 60 \mathrm{~cm} / 30 \mathrm{~cm})$ target instead of a 2-cell system has demonstrated that the false asymmetries can be reduced by a factor $\approx 10$. As shown in Fig. $3 b$ this new configuration presently used allows to have simultaneously the 2 configurations obtained before and after a microwave polarization reversal. Therefore it is enough to rotate the magnetic field only once a day instead of 3 times previously.

A new microwave cavity has been designed and constructed at CERN to provide an efficient polarization and to match the larger solenoid acceptance. A study was performed to determine the spatial microwave uniformity. The effect of the frequency modulation of a microwave field on the level of polarization and built-up time for ${ }^{6} \mathrm{LiD}$ material was also investigated [5]. This study shows that the number of resonant excited modes increases with the diameter of the cavity within the range of frequency [70200 - 70300] $\mathrm{MHz}$ used to polarize ${ }^{6} \mathrm{LiD}$ at $2.5 \mathrm{~T}$. It was demonstrated that the frequency modulation of $10 \mathrm{MHz}$, which is used to improve the speed of polarization of the target material [6], is covering a density of about 4 modes all along the frequency range.

The new cavity is $127 \mathrm{~cm}$ long for a $40 \mathrm{~cm}$ diameter and made of copper. As shown in Fig. 4, this cavity is composed of 3 subcavities along the mixing chamber of the dilution refrigerator following the position of each target cell. Each subcavity is separated from the next or/and previous one by a microwave stopper of $5 \mathrm{~cm}$ length made also of copper and totally microwave leaktight. The microwaves are propagated from the generators to 


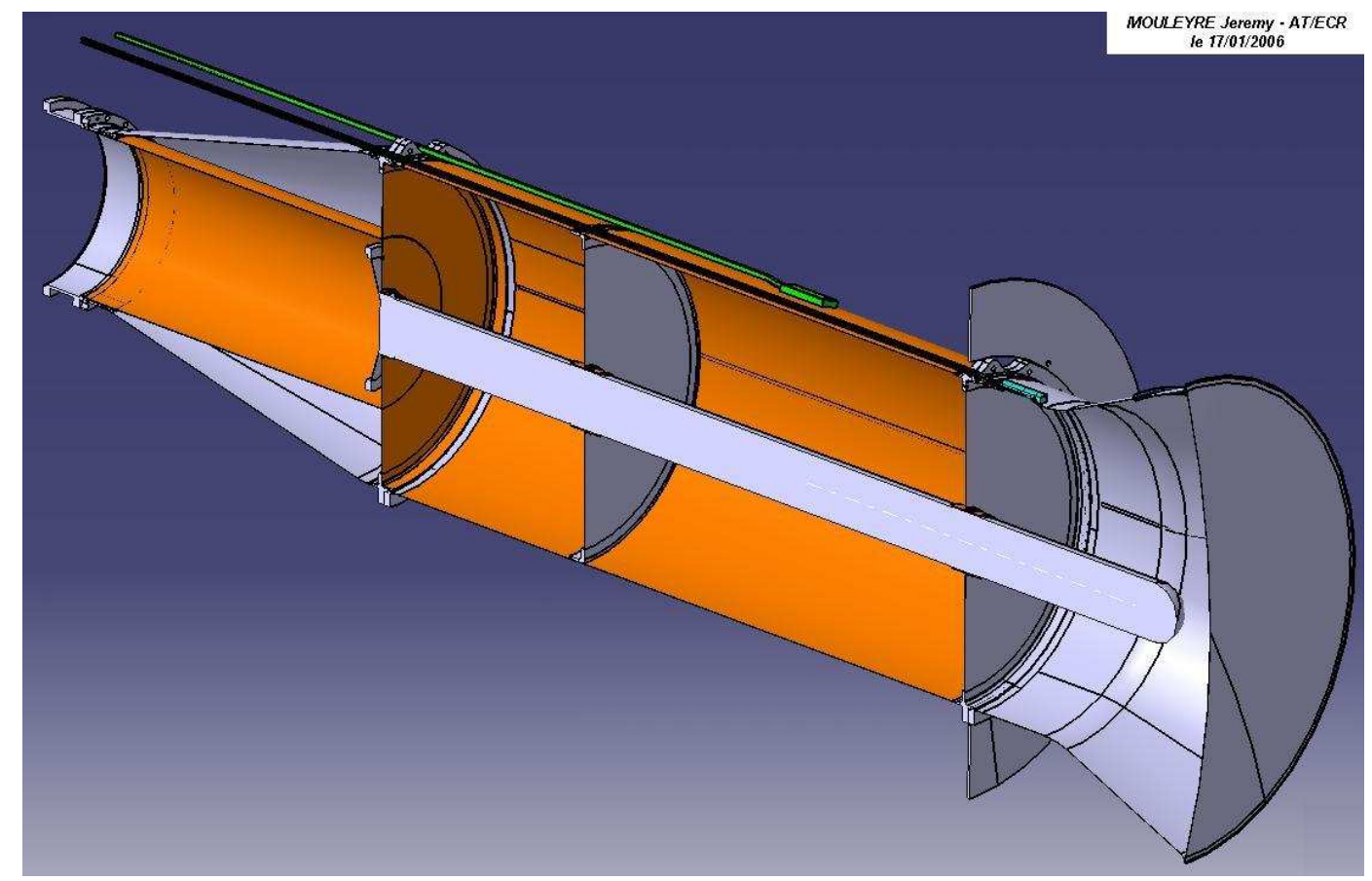

FIGURE 4. New microwave cavity for the new 3-cell polarized target

the cavity through a set of 3 waveguides which are coupled to the cavity by a single hole for each cell [5]. The body of the cavity is cooled through a liquid helium circulation loop maintaining it at $5 \mathrm{~K}$ in the steady state conditions. During a fast discharge the temperature rises up to $55 \mathrm{~K}$ which is still low enough to keep the target material in safe condition.

\section{PRELIMINARY RESULTS AND OUTLOOK}

The assembly of the upgraded target including the larger acceptance magnet, the 3-cell target and the new microwave cavity took place in May 2006. After leak and vacuum tests the target system was cooled down and the 3 -cell target was loaded with ${ }^{6} \mathrm{LiD}$. The very first polarization of the target material was quite encouraging since after several days of DNP a very high average degree of polarization of $+54.0 \%$ (upstream cell), $52.6 \%$ (central cell) and 56.2\% (downstream cell) was reached. The Fig. 5 shows the polarizations performances obtained compared with those from 2004. It is important to notice that the dilution refrigerator was not yet fine tuned during this first polarization build-up. The very good preliminary results are mainly due to the perfect microwave leaktightness between the cells and excellent running conditions of the refrigerator reaching $55 \mathrm{mK}$, a slightly lower temperature than in 2004. 


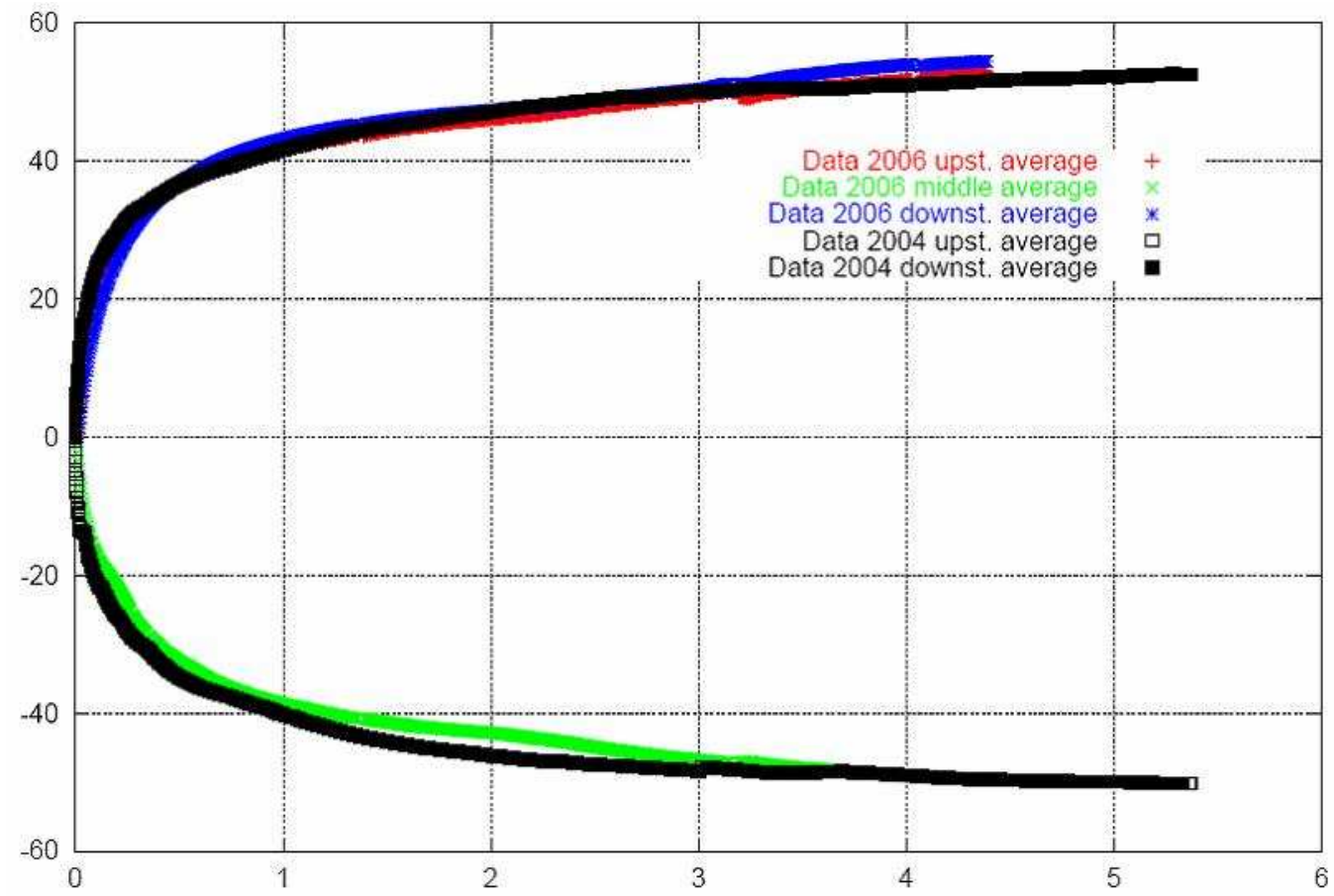

FIGURE 5. preliminary polarisation results of 2006 compared with those of 2004

\section{REFERENCES}

1. COMPASS Collaboration, CERN/SPSLC 96-14 (1996)

2. St. Goertz, The dynamic nuclear polarization process. NIM A526 (2004) 28-42

3. K. Kondo et al., Polarization measurement in the COMPASS polarized target. NIM A526 (2004) 70-75

4. J.F. Gournay, Quench data acquisition and slow control for the superconducting magnet of the COMPASS experiment. ICALEPCS 2005

5. Yu. Kisselev, The modulation effect on the dynamic polarization of nuclear spins NIM A356 (1995) 99-101

6. Yu. Kisselev et al. Investigation of the multimode cavity for the COMPASS magnet, in PST05 conference (2005), Kyoto, to be published 